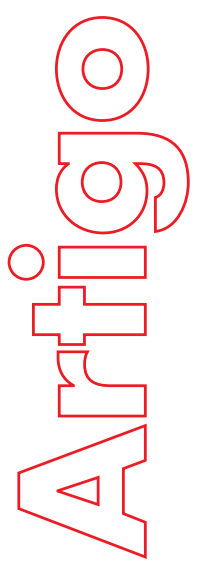

\title{
INFRAESTRUTURAS DE DADOS ESPACIAIS - IDES: PERSPECTIVA ACADEMICA - DESAFIOS E PROPOSTA
}

\author{
José Alberto Quintanilha \\ Cláudia A. Soares Machado
}

\section{Revista}

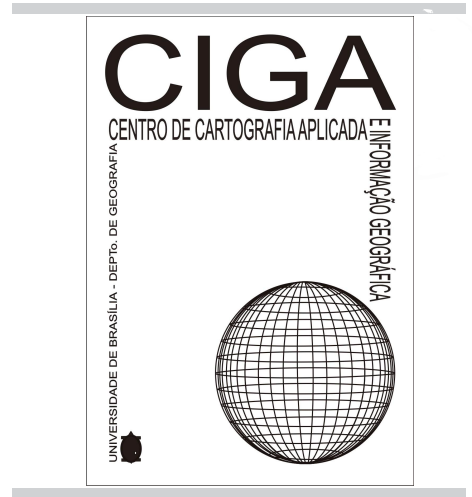

Revista Eletrônica:

Tempo - Técnica - Território, V.9, N.2 (2018), 49:58

ISSN: $2177-4366$

\section{Como citar este ar tigo}

Quintanilha, J.A.; Machado, C.A.S.; INFRAESTRUTURAS DE DADOS

ESPACIAIS - IDES: PERSPECTIVA ACADÊMICA - DESAFIOS E PROPOSTA.

Revista Eletrônica: Tempo - Técnica - Território, v.9, n.2 (2018), p. 49:58 ISSN: 2177-4366.

Disponível em:

http://periodicos.unb.br/index.php/ciga/issue/view/1905

Este obra está licenciado com uma Licença Creative Commons Atribuição - Não Comercial 4.0 Internacional. 


\title{
INFRAESTRUTURAS DE DADOS ESPACIAIS - IDES: \\ PERSPECTIVA ACADÊMICA - DESAFIOS E PROPOSTA
}

\author{
José Alberto Quintanilha \\ Escola Politécnica da Universidade de São Paulo \\ Departamento de Engenharia de Transportes \\ Professor Associado Sênior \\ Doutor em Engenharia de Transportes \\ Mestre em Sensoriamento Remoto \\ Bacharel em Estatística \\ jaquinta@usp.br \\ Cláudia A. Soares Machado \\ Escola Politécnica da Universidade de São Paulo \\ Departamento de Engenharia de Transportes \\ Pós Graduanda \\ Doutora em Engenharia de Transportes \\ Mestre em Engenharia de Transportes \\ Engenheira Civil \\ claudia.machado@usp.br
}

\begin{abstract}
RESUMO: O principal objetivo deste artigo é prover um quadro geral das chamadas IDEs (Infraestruturas de Dados Espaciais) Acadêmicas ou Universitárias e a inclusão de IDEs Acadêmicas Locais como nós em uma infraestrutura de dados espaciais mais geral e abrangente (acadêmica ou não). O artigo foi elaborado a partir das proposições da Dr. ${ }^{a}$ Claire Ellul (University College of London - UCL) e do Dr. Clodoveu Davis Jr (Universidade Federal de Minas Gerais UFMG) e do trabalho de Castelein et al. (2010). Conclui-se que há um entendimento comum sobre a diferença de qualidade dos dados gerados e dos metadados referentes a dados espaciais produzidos na academia. Propõe-se também, que as entradas para uma IDE Acadêmica devam ser tratadas essencialmente como informação voluntária e a criação de um projeto amplo, objetivando o estabelecimento de uma IDE Acadêmica de modo a permitir a sua integração em uma plataforma SIG (Sistema de Informações Geográficas).
\end{abstract}

Palavras chaves: IDE Acadêmica; INDE; SIG; Metadados; "Producers”.

RESUMÉN: El principal objetivo de este artículo es proporcionar un marco general de las llamadas IDEs Académicas o Universitarias y una inclusión de IDEs Académicas locales como nodos en una estructura de datos espaciales más amplia y completa (académica o no). El artículo fue elaborado a partir de las proposiciones de la doctora Claire Ellul (Universidad de Londres - UCL) y del Dr. Clodoveu Davis Jr (Universidad Federal de Minas Gerais - UFMG) y del trabajo de Castelein et al. (2010). Se concluye que hay un consenso sobre la diferencia de calidad de los datos generados y de 
los metadatos sobre datos espaciales producido en la academia. Se propone, también, como entradas para una IDE Académica deban ser tratadas como voluntarias y la creación de un proyecto amplio, con el objetivo de establecer una IDE Académica para permitir su integración en una plataforma SIG (Sistema de Información Geográfica).

Palabras llaves IDE Académica; INDE; SIG; Metadatos; “Producers”.

ABSTRACT: The main objective of this paper is to provide an overview of the Academic SDI (Spatial Data Infrastructure) and an inclusion of Academic SDI as nodes in a more comprehensive spatial data infrastructure (academic or non-academic). The article was elaborated from the propositions of the Dr. Claire Ellul (University of London - UCL) and Dr. Clodoveu Davis Jr (Federal University of Minas Gerais - UFMG) and the work of Castelein et al. (2010). We conclude that there is a general agreement about the difference in terms of the quality of the generated data and the metadata about spatial data produced in the academy. It is also proposed that the inputs for an Academic SDI should be treated as volunteer information and the creation of a broad project, aiming the establishment of an Academic SDI in order to allow its integration into a GIS (Geographic Information System) platform.

Keywords: Academic SDI; INDE; GIS; Metadata; “Producers”.

\section{Introdução}

1.1. Visão geral de uma IDE

Segundo Davis Jr (2016):

“... os princípios básicos de IDEs são: compartilhar o máximo possível; tornar dados e serviços completamente acessíveis (interoperabilidade); usar formatos abertos; ter acesso aos dados nas suas origens/fontes; ter acesso a dados atualizados e ter a garantia de que os dados sejam “legíveis"”.

Ainda segundo Davis Jr. (2016), algumas das perspectivas atuais de uma IDE estão na incorporação de novos componentes tecnológicos de informação (geocodificação, gazetteer, VGI - Volunteered Geographic Information, cloud computing, big data) e num melhor suporte semântico para o catálogo de metadados.

\subsection{Visão geral de uma IDE Acadêmica}

Uma modelagem genérica de uma IDE Acadêmica pode ser vista na Figura 1. 
Modelagem de IDE Acadêmica

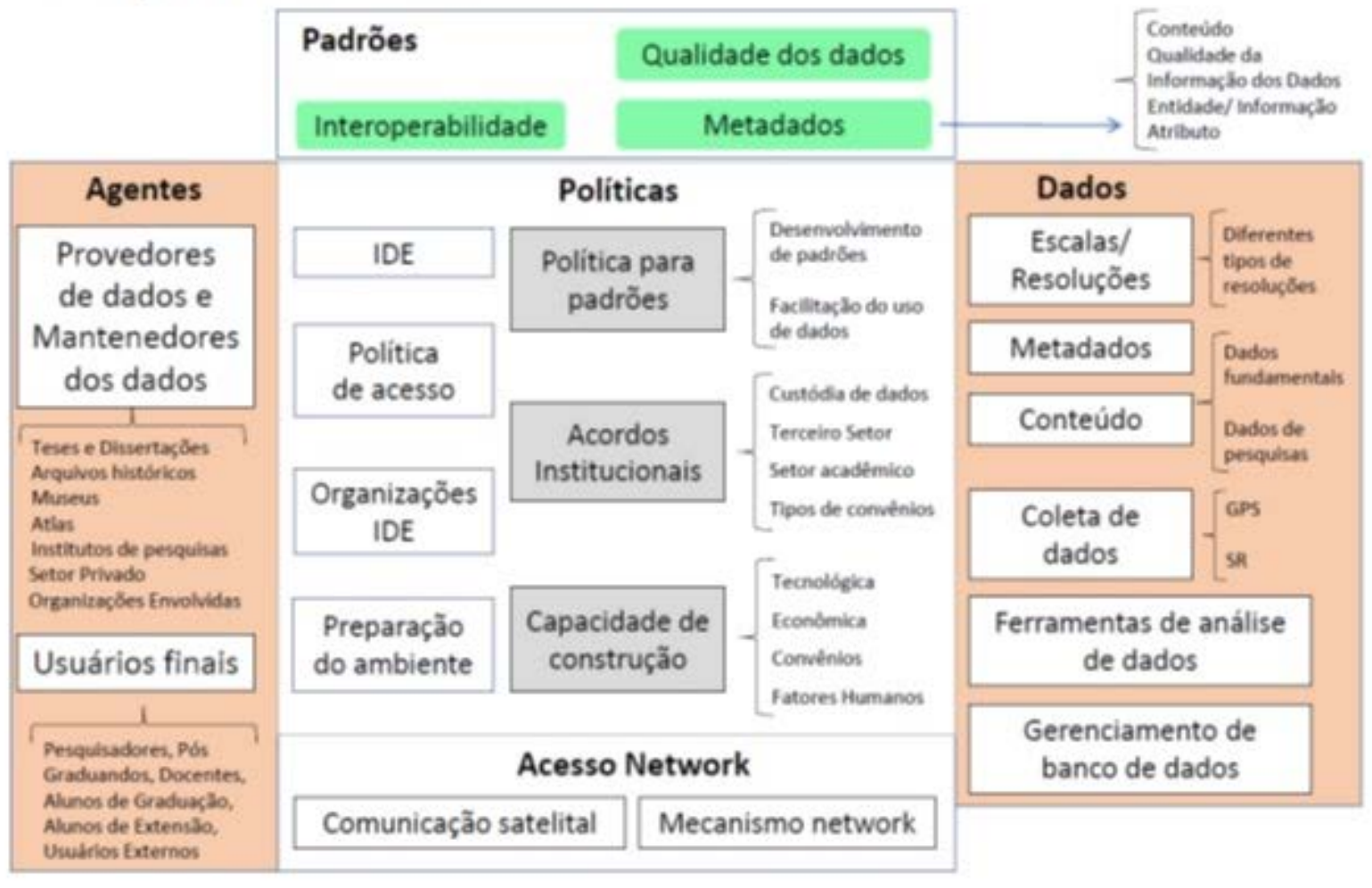

Figura 1: Modelagem de IDE Acadêmica (adaptado de Mansourian et al., 2006 e Silva, 2015).

Nessa Figura, entre os Provedores e Mantenedores de Dados tem-se: as Tese e Dissertações (onde se podem incluir os artigos e relatórios de Iniciação Científica); os Arquivos Históricos sob custódia das universidades, faculdades e museus, dentre outros, assim como o Setor Privado e outras Organizações (Organizações Não Governamentais - ONGs, por exemplo), através dos projetos de Pesquisa \& Desenvolvimento (P\&D).

Dentre os usuários, estão alunos, pesquisadores, professores (inclusive em sala de aulas) e usuários externos ao meio acadêmico.

Em relação aos demais aspectos, há que se considerar que em muitos trabalhos acadêmicos o dado original não pertence às Universidades, nem mesmo quando levantado/obtido por alunos e pesquisadores, como por exemplo, em projetos de Pesquisa \& Desenvolvimento ou de cooperação com órgãos públicos. Por outro lado, os produtos das análises pertencem às Universidades, e são de responsabilidade destas. Isso cria um aspecto importante dentro do que se chama de POLİTICAS e que deve ser tratado de forma diferenciada caso a caso.

Uma comparação entre os metadados tradicionais e os requisitos de uma IDE Acadêmica para uma pesquisa interdisciplinar pode ser vista em Ellul et al. (2017). A maioria dos itens se refere aos metadados (conteúdo, qualidade/adequação a padrões, tempo investido na elaboração, por exemplo), mas também destacam que uma IDE “tradicional” é essencialmente orientada a dados Revista Eletrônica: Tempo - Técnica - Território, V.9, N.2 (2018), 49:58 ISSN: 2177-4366 
espaciais, centrada no produtor de dados e produzida por quem sabe a importância dos metadados, enquanto uma IDE “Acadêmica” trabalha com dados de relatórios e questionários, por exemplo, além do dado espacial e é dirigida aos produtores e usuários, chamados de "producers".

A Lei de Acesso à Informação (Lei 12.527/2001 <http://www.planalto.gov.br/ccivil_03/_ato20112014/2011/lei/l12527.htm> e, no caso de São Paulo, o Decreto Estadual no. 58.052/2012 <http://www.legislacao.sp.gov.br/legislacao/dg280202.nsf/5fb5269ed17b47ab83256cfb00501469/0 d8cf8dcbd4ef45f83257a010046ef75?OpenDocument\&Highlight=0,58.052>), que regulam o acesso à informação previsto na Constituição Federal, também induz que mais órgãos públicos, incluindo universidades e outras instituições de ensino e pesquisa, venham a aderir à INDE - Infraestrutura Nacional de Dados Espaciais e a outras IDEs temáticas, incluindo as acadêmicas (adaptado de Clodoveu Davis Jr., 2016).

\subsection{Objetivos deste artigo}

O principal objetivo deste artigo é prover um quadro geral das chamadas IDEs Acadêmicas ou Universitárias contendo mapas e imagens resultantes de Dissertações, Teses, artigos acadêmicos e relatórios de Iniciação Científica e a de inclusão das IDEs Acadêmicas locais como nós de uma IDE mais geral e abrangente, acadêmica ou não. O artigo foi elaborado a partir da apresentação (Quintanilha e Machado, 2017) feita no evento GeoNordeste de 2017, realizado em Salvador Bahia.

\section{Revisão bibliográfica}

2.1. Versões de uma IDE acadêmica (adaptado de Ellul, 2017)

Ellul (2017) apresenta e discute as seguintes perspectivas relativas a uma IDE Acadêmica:

- Perspectivas na pesquisa

No caso da pesquisa acadêmica há que se considerar alguns aspectos peculiares como a necessidade de se ter acesso a outras IDEs institucionais (no caso brasileiro, por exemplo, a INDE, o DataGEO, etc.), uma vez que muitas vezes as pesquisas são isoladas ou sem continuidade, e que nem sempre o pesquisador ou instituição é “proprietário” do dado, mas apenas dos seus resultados, que podem ser incorporados a IDEs, acadêmicas ou outras.

De todo modo, os resultados devem ter compatibilidade e fazer uso de padrões comuns à IDE que pretende se vincular. Em qualquer caso, há que se considerar, conforme Ellul (2017), possíveis resultados isolados; eventuais usuários não especializados e/ou externos; a qualidade dos dados (usados e gerados) e a adequação dos metadados.

- Perspectivas no ensino 
Ainda segundo Ellul (2017), as salas de aula e laboratórios são potenciais usuários de IDEs Acadêmicas e dos possíveis resultados isolados Nesses casos é preciso saber:

$\checkmark$ Os metadados serão o problema?

$\checkmark$ Deverão ser espaciais?

$\checkmark$ Como decidir o que usar?

$\checkmark$ Com ou sem IDE?

$\checkmark$ Atualização espacial ou temporal ou ambas?

- Perspectivas de pesquisa sobre data curation

Ellul (2017) expõe sua preocupação com o que chama de data curation, que é o gerenciamento de dados ao longo de seu ciclo de vida, desde sua criação e armazenamento inicial até o momento em que é arquivado para posteridade ou tornar-se obsoleto e ser excluído. O objetivo principal do data curation é garantir que os dados sejam recuperáveis de forma confiável para futuros objetivos de pesquisa ou reutilização ${ }^{1}$. Um aspecto importante é o metadado, que deve ser bem entendido, ou seja: (1) Quais dados de quais pesquisas existem? (2) Porque, quando e como foram gerados? (3) Como acessá-los? Outros aspectos passam por uma política de disseminação de dados: (4) Por quanto tempo ficará disponível? (5) Com que qualidade? (6) Qual a obsolescência prevista para o dado?

- Desafios de pesquisa

Vandenbroucke et al. (2012), em relatório relacionado ao INSPIRE (Infrastructure for Spatial Information in the European Community) fazem duas recomendações para melhoria na implementação de IDEs: i) colocar mais ênfase no compartilhamento dos dados, independentemente da forma ou formato destes, ou seja, torná-los disponíveis e acessíveis de forma que a conformidade com as especificações técnicas não sirva de desculpa ou entrave para o compartilhamento; e ii) melhorar o canal de comunicação e entendimento das diversas comunidades que estão ligadas as IDEs. Essa última recomendação aponta a necessidade de se entender melhor os usuários, o compartilhamento de informação ou mesmo o uso e descoberta de serviços que continuam sendo discutidos dentro da comunidade de IDEs (Borba, 2017). No caso especifico da IDE Acadêmica, além dos tópicos indicados pelos autores, há que se considerar os aspectos considerados nos itens 2.2, 2.3 e 2.4 a seguir e, se realmente uma IDE Acadêmica deve incluir outros dados além dos espaciais, como indicado tanto por autores nacionais quanto internacionais.

\subsection{Metodologia de SIG apoiado em IDE}

${ }^{1}$ Fonte: http://whatis.techtarget.com/definition/data-curation) 
Segundo Davis Jr. (2016), sem a existência de IDEs, o uso de dados em Sistemas de Informações Geográficas (SIG), normalmente é baseado em dados inconsistentes em termos de formato e conteúdo; na existência de dados “invisíveis”, em formato não digital ou em formato não editável (por exemplo: “.pdf”), ou escondidos em computadores pessoais; existem todas as dificuldades inerentes de se integrar dados e sistemas; há uma quase completa ausência de descrições de metadados, e as poucas que existem são ruins; e quase nenhum uso de padronização.

No caso de SIGs se valendo de IDEs, ainda segundo Davis Jr. (2016), utiliza-se dados de diferentes fontes externas, dados possivelmente superpostos/conflitantes/redundantes, provenientes de fontes e sistemas de confiabilidade variável (qualidade dos dados), com o adequado versionamento e comportamento temporal, com restrições de integridade distribuídas e com o projeto físico e a escalabilidade já adequados.

Considerando o acima exposto, como ficaria a etapa modelagem de dados de um SIG? No caso particular de uma IDE Acadêmica que teria mais produtos de análises do que dados brutos, sem controle da modelagem, como isso poderia ser tratado?

\subsection{Crowdsourcing e Informação Geográfica Voluntária - VGI (adaptado de Borba, 2017)}

Segundo Borba (2017), “uma área que se beneficiou do uso de crowdsourcing foi justamente aquela que trata do domínio da informação geoespacial. O termo Informação Geográfica Voluntária (ou em inglês Volunteered Geographical Information - VGI) denota informação geográfica que é coletivamente contribuída por uma multidão heterogênea de voluntários (Rehrl et al., 2013). Goodchild (2007) introduziu esse termo para designar o papel da sociedade como produtora de informação geográfica, uma função que durante séculos tem sido executada por instituições oficiais de governo. Também é comum o uso do termo Crowdsourcing Geospatial conforme visto em Heipke (2010); Goodchild e Glennon (2010); Haklay et al. (2014). Assim, VGI também é considerado um tipo de Crowdsourcing. Um fato importante ressaltado por Goodchild (2007), além da heterogeneidade, é a questão do envolvimento generalizado de um grande número de cidadãos voluntários, muitas vezes com pouca qualificação formal, na criação de informação geográfica. Ainda de acordo com este autor, VGI se encaixa no modelo de uma IDE, facilitando a troca de informação geográfica entre os indivíduos em uma comunidade (Goodchild, 2007), apesar das diferenças entre VGI e IDE (Castelein et al., 2010).”, conforme mostrado na Tabela 1.

Componentes de IDE Diferenças

VGI IDE 


\begin{tabular}{lll}
\hline Políticas & $\begin{array}{l}\text { Comunidade de } \\
\text { usuários registrados }\end{array}$ & Organizações formais \\
Redes de acesso & Bidirecionais & Foco uni-direcional \\
Padrões & Padrões de dados & $\begin{array}{l}\text { Padrões de metadados, dados } \\
\text { e serviços }\end{array}$ \\
Dados & $\begin{array}{l}\text { Foco específico ou } \\
\text { Temático }\end{array}$ & Amplo escopo de dados \\
Pessoas & $\begin{array}{l}\text { Amplo conjunto de usuários } \\
\text { não-especializados }\end{array}$ & $\begin{array}{l}\text { Conjunto limitado de usuários } \\
\text { especializados }\end{array}$ \\
\hline
\end{tabular}

Tabela 1: Diferenças entre características de VGI e de IDE (traduzido e adaptado de Castelein et al., 2010).

Em Foody et al. (2017), o capítulo 12 (Demetriou et al., 2017) discute os desafios de se integrar VGI à Infrastructure for Spatial Information in the European Community (INSPIRE); quais são os aspectos mais críticos dessa integração e apresenta um esquema conceitual que integra IDEVGI a uma plataforma SIG. Argumentam que, potencialmente, todos os usuários podem ter benefícios, mas que não é uma tarefa fácil, por conta dos aspectos técnicos e institucionais envolvidos. Sugerem que os próximos passos sobre esse tema sejam focados no estabelecimento de uma ampla rede envolvendo todos os usuários identificados, de maneira a:

“... criar uma estrutura robusta que abranja todos os aspectos de um projeto, desde a concepção inicial até sua implementação, a fim de alcançar exemplos bem-sucedidos de integração e, idealmente, uma plataforma SIG integrada.”

\subsection{Experiências e propostas nacionais}

Um primeiro e importante aspecto sobre as experiências e propostas nacionais de uma IDE Acadêmica, é de que há muito pouca coisa documentada e publicada, à semelhança das experências internacionais, onde há uma concentração de publicações em 2017.

A melhor descrição e documentação localizada até o momento se refere a IDE Acadêmica da UFPR - GeoNode (http://www.idea.ufpr.br/), que é uma plataforma open source para compartilhamento de dados geoespaciais, mapas e documentos. Sobre ela e seu desenvolvimento, existe ao menos uma Dissertação de Mestrado publicada (Fronza, 2016) e dois artigos em eventos (Brito et al. 2014 e Machado et al., 2016).

Nesse artigo, os autores colocam que:

“Contudo, a partir da $2^{a}$ Jornada INDE/Academia, em 2014, as universidades passaram a se organizar no seu papel de também produtoras de dados espaciais, de 
modo que as mesmas deveriam ser integradas a INDE, constituindo mais um nó dentro da infraestrutura existente" (Brito et al. 2014).

Portanto, é recente a tentativa de organização das universidades e ainda não de forma incisiva, justificando a ausência de outros documentos publicados. Preconizam que deve haver a integração das instituições governamentais, acadêmicas, privadas e sociais e a adesão à INDE possibilita tal integração.

“No entanto, mais do que um nó tradicional da INDE, uma IDE Acadêmica possui características únicas que precisam ser consideradas” (Machado et al., 2016).

E, de novo, a abrangência além do dado espacial e os metadados são colocados como diferenças. Ressaltam, entretanto:

“A importância da implantação de um nó da INDE na academia é a otimização dos recursos existentes, proporcionando à comunidade um ambiente colaborativo para o compartilhamento de dados” (Machado et al., 2016).

\section{Metodologia}

O artigo foi elaborado a partir das proposições de Claire Ellul (University College of London UCL) e Clodoveu Davis Jr. (Universidade Federal de Minas Gerais) e do trabalho de Castelein et al. (2010).

No primeiro caso, a Profa. Claire Ellul estabelece uma estrutura para a abordagem aqui adotada de IDEs Acadêmicas, e o Prof. Clodoveu Davis Jr apresenta questões referentes à utilização de IDEs em geral, por sistemas de informações geográficas, e que aqui foram adaptadas ao conceito adotado de IDE Acadêmica.

Castelein e parceiros apresentam uma comparação entre VGI e IDE, ambos tratados como crowdsourcing, e que aqui foi adotada para uma proposta de inserção de dados em IDEs acadêmicas.

Finalmente, a sugestão de inserção das IDEs Acadêmicas locais como nós de uma IDE mais geral e abrangente, acadêmica ou não, como já comentado por Brito (2014), surge da dificuldade atual tanto para a captação de recursos financeiros para o desenvolvimento de IDEs, quanto da identificação de pessoal capacitado em quantidade suficiente para atender as necessidades demandadas, dadas as dimensões do país, quantidade de universidades e produtos gerados, e as atuais condições tecnológicas (redes, clusters, nuvem, etc.).

\section{Constatações}

4.1. Qual a real utilidade dos metadados? Quem cria/usa metadados e como?

4.2. Ajustar às formas de como as pessoas trabalham (com IDE). 
4.3. Estudantes (só eles?) são pressionados a cumprir prazos e geram versões resumidas, difíceis de serem entendidas por possíveis usuários.

4.4. Uso para ensino em outras disciplinas.

4.5. Onde encontrar financiamento para pesquisa?

\section{Conclusões}

5.1. Qualidade (nos seus diferentes aspectos) dos dados disponíveis numa IDE, principalmente as de caráter acadêmico.

5.2. As entradas para uma IDE Acadêmica não deveriam ser tratadas essencialmente como VGIs?

5.3. A necessidade de se discutir o grau e a importância da familiaridade que um potencial usuário de uma IDE (Ellul et al., 2012), em particular a(s) acadêmica(s), deve ter com metadados e da sua inclusão no processo de aderência à IDE, principalmente para aqueles produtos que podem ser diretamente utilizados em pesquisas posteriores ou de caráter interdisciplinar.

5.4. Discutir a adoção (ou não) da proposta europeia apresentada no item 2.3 e, além da rede já estabelecida e ainda em desenvolvimento envolvendo todos os usuários identificados, se criar um projeto amplo, objetivando o estabelecimento de uma IDE Acadêmica de modo a permitir a sua incorporação a uma plataforma SIG integrada.

\section{Agradecimentos}

Os autores agradecem à Escola Politécnica da Universidade de São Paulo, ao Conselho Nacional de Desenvolvimento Científico e Tecnológico (CNPq), e à Fundação para o Desenvolvimento Tecnológico da Engenharia (FDTE) pelo apoio institucional e suporte financeiro para a condução deste trabalho.

\section{Referências}

BORBA, R. L. R. Ecossistema para infraestrutura de dados espaciais híbrida, coproduzida, colaborativa, convergente e compartilhável - Tese de Doutorado - Programa de Pós-graduação em Engenharia de Sistemas e Computação, COPPE, Universidade Federal do Rio de Janeiro, Engenharia de Sistemas e Computação. Rio de Janeiro, RJ, 2017.

BRITO, P. L. A Universidade como nó da INDE. Palestra proferida na Segunda Jornada INDE Academia. Brasília: Ministério do Planejamento, 27 e 28 de maio de 2014. 
BRITO, P. L.; SOUZA, F. A.; CAMBOIM, S.; GIANNOTTI, M. A. Primeiros passos para a implementação de uma IDE Universitária. V Simpósio Brasileiro de Ciências Geodésicas e Tecnologias da Geoinformação. Recife, PE, 2014, p. 77 - 84.

CASTELEIN, W.; GRUS, L.; CROMPVOETS, J.; BREGT, A. A characterization of volunteered geographic information. 13th AGILE International Conference on Geographic Information Science 2010. Guimarães, Portugal. 10 p.

DAVIS JR., C. Infraestruturas de Dados Espaciais: aplicações na Web e perspectivas. Apresentação no Workshop IDE para acidentes envolvendo o transporte rodoviário de produtos perigosos (TRPP), Escola Politécnica da Universidade de São Paulo, São Paulo, SP, 2015.

DEMETRIOU, D.; CAMPAGNA, M.; RACETIN, I.; KONECNY. M. Chapter 12: Integrating Spatial Data Infrastructures (SDIs) with Volunteered Geographic Information (VGI) for creating a Global GIS platform. In: FOODY, G, et al. (eds.). Mapping and the Citizen Sensor. London: Ubiquity Press, 2017.

ELLUL, C. Improving the Usability of Geospatial Data - An Academic Perspective. University College London. 2017. <http://www.eurogeographics.org/sites/default/files/Claire\%20Ellul\%20Eurogeographics\%20Prese ntation\%2014th\%20June\%202017.pdf>

ELLUL, C.; WINER, D.; MOONEY, J.; FOORD, J. Chapter4: Bridging the Gap between Traditional Metadata and the Requirements of an Academic SDI for Interdisciplinary Research, pp.57- 78. In RAJABIFARD, A.; COLEMAN, D. (eds). Spatially Enabling Government, Industry and Citizens. Global Spatial Data Infrastructure (GSDI) Association Press, 2012, 277p.

FOODY, G; SEE, L; FRITZ, S; MOONEY, P; OLTEANU-RAIMOND, A-M; FONTE, C. C.; ANTONIOU, V (eds.). Mapping and the Citizen Sensor. London: Ubiquity Press, 2017.

FRONZA, G. IDE Acadêmica: construção de uma infraestrutura de dados espaciais colaborativa. Dissertação (Mestrado) em Ciências Geodésicas, no Curso de Pós-Graduação em Ciências Geodésicas, Setor de Ciências da Terra, Universidade Federal do Paraná, Curitiba, 2016.

GOODCHILD, M. F. Citizens as voluntary sensors: spatial data infrastructure in the world of Web 2.0. International Journal of Spatial Data Infraestructures Research, v. 2, 2007.

GOODCHILD, M. F.; GLENNON, J. A. Crowdsourcing geographic information for disaster response: a research frontier. International Journal of Digital Earth, v. 3, n. 3, 2010, p. 231-241. 
HAKLAY, M. E.; ANTONIOU, V.; BASIOUKA, S.; SODEN, R.; MOONEY, P. Crowdsourced Geographic Information Use in Government. Report to GFDRR (World Bank Publications). UCLGeomatics, London, 2014.

MACHADO, A. A.; DA SILVA, E. S.; FRONZA, G.; DE CAMPOS, R. G.; FERRI, K. C.; PISETTA, J. A.; CAMBOIM, S. P. Projeto e implementação de uma IDE Acadêmica na UFPR. IX Colóquio Brasileiro de Ciências Geodésicas. UFPR, 2016. 14 p.

MANSOURIAN, A.; RAJABIFARD, A.; ZOEJ, M. V.; WILLIAMSON, I. Using SDI and webbased system to facilitate disaster management.Computers \& Geosciences, v.32, n.3, 2006, p.303315.

QUINTANILHA, J. A.; MACHADO, C. A. S. "IDEs: perspectiva acadêmica". Apresentação no evento GeoNordeste 2017 - Seção Técnica da Rede IDE Acadêmica, Salvador, BA, 2017.

SILVA, J. B. Infraestruturas de Dados Espaciais para TRPP. Apresentação no Workshop IDE para acidentes envolvendo o transporte rodoviário de produtos perigosos (TRPP), Escola Politécnica da Universidade de São Paulo, São Paulo, SP, 2015.

VANDENBROUCKE, D.; CROMPVOETS, J.; BAMPS, C.; JANSSEN, K. D4.2 -Spatial Data Infrastructures in Europe, State of play spring 2011: Summary report regarding the results of the European Assessment of 34 NSDI, 2012. 